\title{
Neural and Behavioral Evidence for Affective Priming from Unconsciously Perceived Emotional Facial Expressions and the Influence of Trait Anxiety
}

\author{
Wen Li ${ }^{1}$, Richard E. Zinbarg ${ }^{1}$, Stephan G. Boehm ${ }^{2}$, and Ken A. Paller ${ }^{1}$
}

\begin{abstract}
Affective judgments can often be influenced by emotional information people unconsciously perceive, but the neural mechanisms responsible for these effects and how they are modulated by individual differences in sensitivity to threat are unclear. Here we studied subliminal affective priming by recording brain potentials to surprise faces preceded by 30-msec happy or fearful prime faces. Participants showed valenceconsistent changes in affective ratings of surprise faces, although they reported no knowledge of prime-face expressions, nor could they discriminate between prime-face expressions in a forced-choice test. In conjunction with the priming effect on affective evaluation, larger occipital P1 potentials at 145$175 \mathrm{msec}$ were found with fearful than with happy primes, and source analyses implicated the bilateral extrastriate cortex in this effect. Later brain potentials at 300-400 msec were
\end{abstract}

\section{INTRODUCTION}

At any given moment we are aware of only a small fraction of the tremendous amount of information impinging on our sense organs. How does sensory processing come to influence everyday behavior without our knowledge? It is not surprising that seeing an intense facial expression on another person can have a strong impact on one's emotional state and social response. On the other hand, it is not obvious how the mere glimpse of a facial expression can have an impact even in the absence of any awareness of viewing the expression. Here we investigate this sort of unconscious influence on social judgments in a subliminal affective priming paradigm with simultaneous recordings of neural responses, in conjunction with analyses of personality factors that may modulate these effects.

Facial expressions are particularly effective in alerting others to impending threat. Threat-related information can be critical for survival and often engages privileged processing, independent of conscious perception (Phelps, 2006; Öhman, 2005; Morris \& Dolan, 2001; Williams, Watts, MacLeod, \& Mathews, 1997). Recently, it has been

\footnotetext{
${ }^{1}$ Northwestern University, ${ }^{2}$ University of Wales Bangor
}

proposed that humans maintain an automatic "default system" that continuously surveys our environment for salient stimuli (Raichle \& Gusnard, 2005; Gusnard \& Raichle, 2001). Similarly, Öhman (1988) conjectured that a completely unconscious detector system automatically scans the environment for threat stimuli. When a stimulus of significant threat is detected by this "quick and dirty" evaluation (LeDoux, 1995), conscious and elaborate threat analysis will occur, with the possible cost of modifying or disrupting other cognitive processing (also see Mathews \& Mackintosh, 1998). These ideas highlight the intriguing notion that our volitional behavior is regularly subject to involuntary affective influences.

Prior demonstrations that unconscious affective information can modulate social behavior have typically involved affective priming paradigms in which subliminal presentations of a prime object shift subsequent affective evaluation of a supraliminal target object in the direction consistent with the prime affect (Fazio, 2001; Murphy \& Zajonc, 1993). A comprehensive understanding of these affective priming effects will require taking the role of individual differences in personality into account. The relevance of personality for affective processing has already been demonstrated in many other situations. For example, individuals prone to social anxiety

enhanced with happy versus fearful primes, which may re-
flect differential attentional orienting. Personality testing for sensitivity to threat, especially social threat, was also used to inal affective priming. Indeed, participants with high trait anxiety demonstrated stronger affective priming and greater
P1 differences than did those with low trait anxiety, and these effects were driven by fearful primes. Results thus suggest that unconsciously perceived affective information influences
social judgments by altering very early perceptual analyses, ple are oversensitive to threat. In this way, perception may be subject to a variety of influences that govern social
preferences in the absence of concomitant awareness of such influences. 
tend to view certain social situations as catastrophic even though others would judge the same situation as neutral (Clark \& Wells, 1995). A pivotal mechanism for such effects and, more generally, for symptoms of clinical anxiety disorders, may be enhanced unconscious processing of threat (e.g., Beck \& Clark, 1997; Mathews \& Macleod, 1994). Studies employing techniques of emotional Stroop or dot-probe interference/facilitation also suggest that anxiety accentuates the influence of subliminal threat on simple cognitive tasks (Mogg \& Bradley, 1998; Williams et al., 1997).

Despite previous demonstrations of subliminal affective priming, a better understanding of the neural underpinnings of this effect is needed. Unconsciously perceived threat may exert its effects via a set of processes such as enhanced sensory processing, a shift in attentional focus, and perhaps a call to action to determine the source of the threat and to execute relevant behavior. To examine and distinguish among these processes, temporally precise neural information is crucial. Therefore, we chose to combine behavioral analyses of subliminal affective priming with recordings of eventrelated potentials (ERPs), which provide on-line measures of neural processing with high temporal resolution.

Prior ERP research has successfully identified enhanced threat processing as early as $80 \mathrm{msec}$ poststimulus (Keil, Moratti, Sabatinelli, Bradley, \& Lang, 2005; Pourtois, Grandjean, Sander, \& Vuilleumier, 2004; Williams et al., 2004; Holmes, Vuilleumier, \& Eimer, 2003; Smith, Cacioppo, Larsen, \& Chartrand, 2003; Eimer \& Holmes, 2002; Pizzagalli et al., 2002). These results converge on the idea that threatening visual stimuli can enhance the occipital or occipitoparietal P1, a visual ERP that peaks around $100 \mathrm{msec}$ poststimulus and that is thought to index early perceptual processing in the extrastriate cortex (Gomez Gonzalez, Clark, Fan, Luck, \& Hillyard, 1994; Mangun, Hillyard, \& Luck, 1993). The idea that these threat-related P1 effects reflect increased extrastriate activity (Pourtois et al., 2004) provides precise temporal information that complements other neuroimaging findings (e.g., Vuilleumier, Armony, Driver, \& Dolan, 2003; Pessoa, McKenna, Gutierrez, \& Ungerleider, 2002; also see Phelps, 2006 for a review). Using an emotional Stroop paradigm, we have recently showed that this P1 enhancement can be elicited by subliminal threat delivered via visual words, and that it increases systemically with people's proneness to anxiety (Li, Zinbarg, \& Paller, in press). We thus predicted that measures of occipital P1 potentials to faces would be sensitive to unconscious affective information and the influence of trait anxiety (TA).

Other potentials may also be sensitive to threat processing. The N2/P3a complex, for example, has been implicated in attentional orienting to emotional stimuli (Cuthbert, Schupp, Bradley, Birbaumer, \& Lang, 2000; Halgren \& Marinkovic, 1995; Johnston, Miller, \& Burleson, 1986). Orienting of attention at these later stages, espe- cially as indexed by $\mathrm{N} 2$ potentials, could be automatic in that it is independent of ongoing cognitive demands (Kenemans, Verbaten, Melis, \& Slangen, 1992). Indeed, Liddell, Williams, Rathjen, Shevrin, and Gordon (2004) demonstrated greater $\mathrm{N} 2$ and faster $\mathrm{P} 3 \mathrm{a}$ in response to threat versus neutral faces presented below conscious awareness. Late positive ERP components (300 msec poststimulus) are also augmented by both positive and negative affect (Simon-Thomas, Role, \& Knight, 2005; Liddell et al., 2004; Schupp, Junghofer, Weike, \& Hamm, 2003; Eimer \& Holmes, 2002; Cuthbert et al., 2000; Dietrich et al., 2000; Schupp et al., 2000). Positive potentials found at 400-500 msec for faces displaying threatening emotions compared to faces with friendly or neutral expressions (Schupp et al., 2004) may also provide evidence for the impact of subliminal facial affect on information processing subsequent to the initial "quick and dirty" analysis (LeDoux, 1995).

A challenging methodological issue in studies of unconscious processing is to ensure that putative subliminal presentations do not lead to conscious awareness of prime stimuli (Pessoa, 2005). In this study, we excluded conscious perception of facial expressions of primes through the following steps: (1) prime faces were presented for 30 msec with backward masking by faces of other individuals; (2) the affect-judgment task did not require processing of prime faces; (3) participants were not informed about prime faces; (4) at the conclusion of the task, any information participants could share about prime faces was gradually revealed in a step-by-step interview; and then, (5) trial-by-trial two-alternative forced-choice tests were administered to assess conscious perception of masked primes.

We produced subliminal priming using prime faces with either fearful or happy expressions. Surprise faces served as masks and as to-be-rated faces. We hypothesized that priming would lead to valence-specific shifts in affective ratings. We reasoned that $\mathrm{P} 1, \mathrm{~N} 2 / \mathrm{P} 3 \mathrm{a}$, and late positive potentials could provide evidence concerning whether early perceptual processing, attention orienting, and late elaborate processing, respectively, are altered in parallel with behavioral priming effects. We also conjectured that TA would modulate behavioral measures of affective priming and ERP correlates of affective priming.

\section{METHODS}

\section{Participants}

Thirty-four right-handed undergraduates were selected from 150 college students based on their scores on the Behavioral Inhibition Scale (Carver \& White, 1994), a measure of TA (detailed below). The sample consisted of 17 students with the highest scores (top 11\%, scores ranged from 24 to 28) and 17 students with the lowest scores (bottom 11\%, scores ranged from 11 to 17). 
Participants had normal or corrected-to-normal vision, and were right-handed. All participants gave informed consent and received class credit for taking part in the experiment.

Two participants chose to skip electroencephalogram (EEG) recordings. One of them and another two participants did not complete the awareness check described below and were excluded from analysis. The awareness check yielded no evidence for conscious perception of emotional expressions of primes among any participants. Thus, we included 31 subjects in behavioral analyses and 30 in ERP analyses.

\section{Questionnaires}

We administered two self-report inventories to measure TA, which is a personality trait concerning sensitivity to threat and proneness to anxiety. A relatively general measure (Behavior Inhibition Scale-see below) was used to select participants to include those with either high or low TA. The second measure (Social Phobia Scale-see below) assesses trait social anxiety, which pertains to sensitivity to social threat in particular. Social anxiety has been linked with processing facial expressions, in that it is associated with enhanced responses to fearful or angry relative to neutral or happy faces (Phan, Fitzgerald, Nathan, \& Tancer, 2006; Killgore \& YurgelunTodd, 2005). Given that priming in our paradigm depends on the processing of facial affect, we suspected that social TA might modify the magnitude of priming and ERP correlates of priming. Scores on these two indices were combined to form a reliable and sensitive measure.

\section{Behavioral Inbibition Scale}

The Behavioral Inhibition Scale (BIS) consists of seven items (e.g., I worry about making mistakes; criticism or scolding hurts quite a bit), designed to assess TA (Carver \& White, 1994). Participants produced a rating for each item on a 4-point scale ranging from 1 (very little) to 4 (very much). The BIS has adequate reliability, with estimates of .74 for the alpha coefficient and .66 for the 8-week test-retest reliability (Carver \& White, 1994). The alpha coefficient assessed in this study was .85 .

The choice of BIS as the measure of TA in this study stemmed from assertions made by Carver and White (1994) and Fowles (1987) that, when measuring TA, one should focus on the sensitivity of the anxiety system rather than a person's typical or average anxiety level. They note that scales such as the Trait Scale from the State and Trait Anxiety Inventory (Spielberger, 1983) and the Manifest Anxiety Scale (Taylor, 1953) mainly tap average anxiety level (e.g., I feel calm; I am jittery) instead of sensitivity to threat. For instance, people with high anxiety vulnerability may learn to avoid anxietyprovoking situations more readily than others and, consequently, experience relatively little anxiety on a daily basis

The BIS was designed to assess anxious responses to threatening situations instead of general affective tone. Studies among large samples of psychiatric patients $(n>1800)$ have demonstrated the validity of the BIS as a measure of TA (Campbell-Sills, Liverant, \& Brown, 2004; Johnson, Turner, \& Iwata, 2003). Also, a recent neuroimaging study showed that the BIS predicted neural responses to fearful pictures (Mathews, Yiend, \& Lawrence, 2004).

\section{Social Phobia Scale}

The Social Phobia Scale (SPS) is used to measure sensitivity to social threat (Mattick \& Clarke, 1998). The SPS consists of 20 items expressly pertinent to social situations (e.g., I become anxious if I have to write in front of other people; I am worried people will think my behavior odd). Participants rated their general patterns concerning each statement with a 5-point scale ranging from 0 (very little) to 4 (very much). The high internal consistency for this measure obtained in this study indicated strong reliability (alpha coefficient $=.83$ ).

At the beginning of the main experimental session, which was 5 to 8 weeks after the initial assessment, participants completed the BIS a second time and the SPS for the first time. BIS and SPS scores collected during the experiment were highly correlated, $r=.62$, $p<.001$. After correction for attenuation due to the imperfect reliability of the measures, the correlation coefficient amounted to .74. We formed a composite measure to tap TA with an emphasis on TA pertinent to social threat by averaging standardized scores of the BIS (at the second assessment) and the SPS. This composite is denoted as the TA measure henceforth in this article. The composite TA score in our sample varied from -1.75 to 2.24 , with a standard deviation of .90 . Analyses on the basis of BIS or SPS scores considered separately produced similar patterns of results for the dependent variables of interest (Appendix I), and the corresponding correlation coefficients with the dependent variables of interest for the two questionnaires did not differ significantly $(p s>.1)$.

\section{Stimuli}

We used surprise faces based on prior experiments that took advantage of the ambiguity of facial expressions of surprise (Kim et al., 2004; Kim, Somerville, Johnstone, Alexander, \& Whalen, 2003). Indeed, outside the laboratory, surprise can result from a happy experience (e.g., a surprise birthday party) or from a fearful experience (e.g., seeing a car crash), so we reasoned that ambiguous expressions of surprise might be sensitive to the influence of subliminal happy or fearful primes. Fearful, 
happy, and surprise faces were obtained from the Karolinska Directed Emotional Faces collection (Lundqvist, Flykt, \& Öhman, 1998). All face images are frontal views, in color, with a consistent background (Figure 1). The test included 140 surprise faces (featuring 70 different people, each showing two surprise expressions), 7 happy faces, and 7 fearful faces. We selected faces showing prototypical happy or fearful emotions from among 140 happy and 140 fearful face photographs. Pleasant ratings (based on a six-point scale from extremely unpleasant to extremely pleasant) from an independent group of 10 undergraduates were significantly different for the two emotion sets $[t(9)=13.17$, $p<.001]$. Faces were presented on a dark background and subtended a visual angle of about $2^{\circ} \times 2.8^{\circ}$.

\section{Procedure}

During the experiment, a participant sat in a dimly lit, sound-attenuated chamber, facing a CRT monitor $140 \mathrm{~cm}$ away. The experimenter and the participant communicated over an intercom.

\section{Affective Judgments}

Each trial was comprised of a fearful or happy face for $30 \mathrm{msec}$ followed by a surprise face for $800 \mathrm{msec}$ (Figure 1A). The 30-msec stimulus onset asynchrony (SOA) is common in related research (e.g., Etkin et al., 2004; Öhman \& Soares, 1998; Whalen et al., 1998), and pilot testing showed that this SOA was sufficiently brief to consistently prevent conscious perception of prime faces (see details below). Each of the 140 surprise faces was presented twice, once with a fearful prime and once with a happy prime. Also, each surprise face was presented once among the initial 140 trials and once among the latter 140 trials. Each fearful and happy prime was presented 20 times. The individual identity of each target face was never the same as that of the preceding prime face. Trials were pseudorandomized and administered in four blocks, with each block consisting of 35 fearful trials and 35 happy trials. Participants were instructed to judge the affect of surprise faces on a bipolar scale ranging from 1 to 6 and corresponding to "extremely positive," "moderately positive," "mildly positive," "mildly negative," and "moderately negative," and "extremely negative," respectively. There was no option for a neutral-affect response, such that each response must be either positive or negative, which may enhance sensitivity for detecting affective priming. Subjects were encouraged to pay close attention to subtle differences in facial affect, and response speed was not stressed. Participants responded using a keypad with buttons in two rows. For half of the participants, the top three buttons were used to indicate negative affect and the bottom three positive affect. Button assignments were reversed for the other participants.

\section{Awareness Check}

At the conclusion of the affective-judgment task, a funnel interview was conducted with five step-by-step questions designed to probe for information about prime perception while only gradually providing information about what actually transpired in the experiment. The five questions were: (1) Did you see anything besides the surprise faces? (2) Did you see anything right before the surprise faces? (3) There was actually a flicker before the surprise faces. What did you see? (4) Did you see a face? (5) What expression did you see in the face?

This interview portion of the procedure served as an initial check for subjective awareness of primes. Seven participants reported seeing some images prior to the
Figure 1. Experimental procedure for the affective judgment task (A) and the awareness check (B).

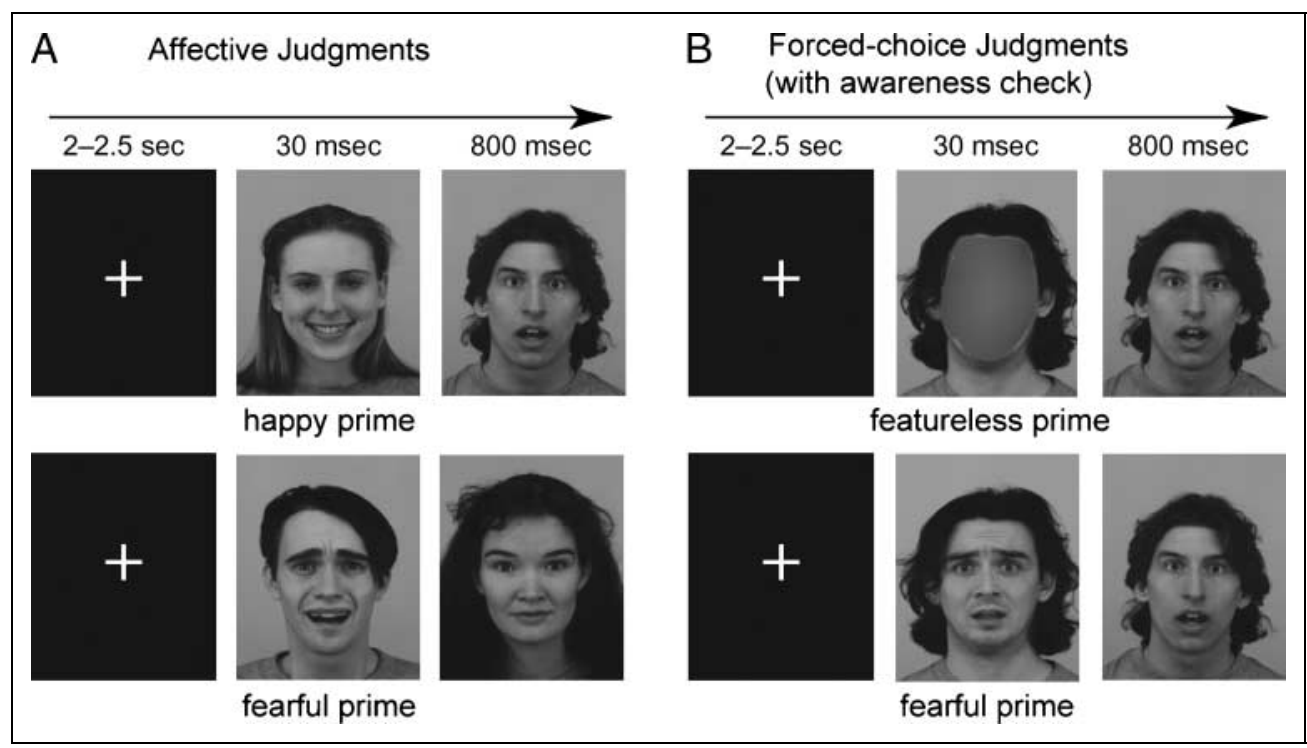


target without knowing the content of these images. Four other participants reported perceiving faces immediately before the target faces but would not volunteer any knowledge about any expressions on these faces. On the basis of these subjective reports, a reasonable inference is that participants were unaware of the affective content of prime faces.

These first-person accounts were supplemented with objective measures of the ability to perceive information concerning prime faces. We included the 14 prime faces used in the affective-judgment task and these same faces with internal facial features blurred (Figure 1B). A total of 56 trials were created, two trials with each of these 28 images, always presented for $30 \mathrm{msec}$, and each time masked by one of the surprise faces. Unlike the affective-judgment task, for this procedure, participants were informed that each surprise face was preceded by another brief stimulus presentation. Participants were instructed to respond first according to whether they thought this prime stimulus was a real face or a featureless face, followed by a rating of confidence on a scale of 1 (minimal confidence) to 3 (strong confidence). Participants then responded according to whether they thought the prime face displayed positive affect or negative affect, again with a confidence rating. Participants were instructed to make a decision on the affect of the prime face even when it was endorsed as featureless. The requirement to rate affect on all trials seemed reasonable to participants because their confidence in face/featureless face discrimination was very low.

Indeed, participants were typically unable to accurately discriminate real faces from featureless faces, with an overall average of 55\% correct (hits plus correct rejections), which was slightly but significantly above the chance level of $50 \%(t=2.37, p=.02)$. Performance in each individual was analyzed to determine whether accuracy ever exceeded the 95\% (one-tailed) cutoff of nonchance performance according to the binomial distribution (which was 61\%; Kemp-Wheeler \& Hill, 1988). This cutoff was exceeded in six participants. Four of these participants exhibited very low confidence scores averaging 1.6, which was the same as that in the group of remaining participants, whereas two showed moderate confidence (mean confidence scores of 2.3 and 2.0; mean accuracy scores of $79 \%$ and $66 \%$, respectively).

As for affect discrimination, individually, none of the participants showed accuracy above the 95\% (onetailed) cutoff for nonchance performance (which was $66 \%$, given that there were half as many relevant trials compared to face/featureless face discrimination). At the group level, the sample mean $(51 \%, S D=12 \%$; adjusted for missed responses) did not significantly exceed chance $(t=.07, p=.94)$. In sum, our multistep awareness check suggests that although a few participants might have some explicit knowledge of the presence of primes, none of the 31 participants had conscious awareness of the facial expressions of primes (the central experimental manipulation in the current study), which allowed us to draw inferences on subliminal affective processing.

\section{EEG Procedures and Data Analysis}

EEG was recorded using a 61-electrode elastic cap plus four eye-movement electrodes (two at the external canthi and two infraorbital). All but the eye electrodes were referenced to the right mastoid. Horizontal eye electrodes were referenced to each other. Infraorbital electrodes were referenced to the corresponding prefrontal electrode positioned above the eyebrows. Impedance was reduced to $5 \mathrm{k} \Omega$. Signals were amplified with a 0.05 to $100-\mathrm{Hz}$ band pass and digitized at $1000 \mathrm{~Hz}$.

EEG and electrooculogram signals were averaged offline for 1000-msec periods starting $200 \mathrm{msec}$ prior to prime onset. ERPs were re-referenced using the average of right and left mastoid recordings. To exclude trials contaminated by artifacts, trials with a voltage exceeding $\pm 75 \mu \mathrm{V}$ at any electrode, relative to the 200 -msec baseline, were discarded before further analysis.

\section{ERP Analysis}

ERPs were averaged separately for fearful and happy trials. An average of 119 (out of 140) trials remained in each condition after artifact rejection. Mean amplitude values were computed for four time intervals, timelocked to prime onset (time 0). The first interval was 145-175 msec, centered on the mean peak latency of the occipital P1 component (Figure 2A). As four occipital electrodes closely surround the Oz scalp location (Figure 2B), we collapsed P1 amplitudes across these electrodes. We also computed mean amplitudes from intervals of 200-300 msec, 300-400 msec, and 400$800 \mathrm{msec}$, corresponding to N2, P3a, and late positive potentials, respectively. These ERP measures were obtained from six midline electrodes, which were grouped into three regions for analysis (Figure 3B).

To analyze relationships between these ERP measures and TA, we treated the composite TA score as a continuous variable in a multiple regression analysis (West, Aiken, \& Krull, 1996). Whereas analysis of variance is a special case of multiple regression in which all the independent variables are categorical, the multiple regression approach has superior power for revealing relationships between TA and ERPs (and also between TA and affective ratings).

\section{Low-resolution Brain Electromagnetic Tomography Analysis}

As a method for estimating cortical sources of ERP effects, we submitted ERP data for intervals showing significant ERP priming effects to low-resolution brain 
Figure 2. (A) ERP waveforms for fearful and happy conditions at an occipital site, with a 200-msec baseline prior to the onset of prime faces. (B) Topography of differential ERPs (fearful-happy) over the interval from 145 to $175 \mathrm{msec}$ The four electrodes where P1 amplitudes were examined are marked by large dark dots. (C) In the LORETA source model, bilateral lingual gyri (marked in black and circled on coronal and sagittal brain images) exhibited significantly greater current density for fearful than happy trials at 145-175 msec, in the high-TA group; $t=4.07$ corresponds to $p=.001$. (D) Trait anxiety correlated with the magnitude of affective priming (left, circular symbols and dotted regression line) and differential P1 amplitude (right, diamond symbols and solid regression line).

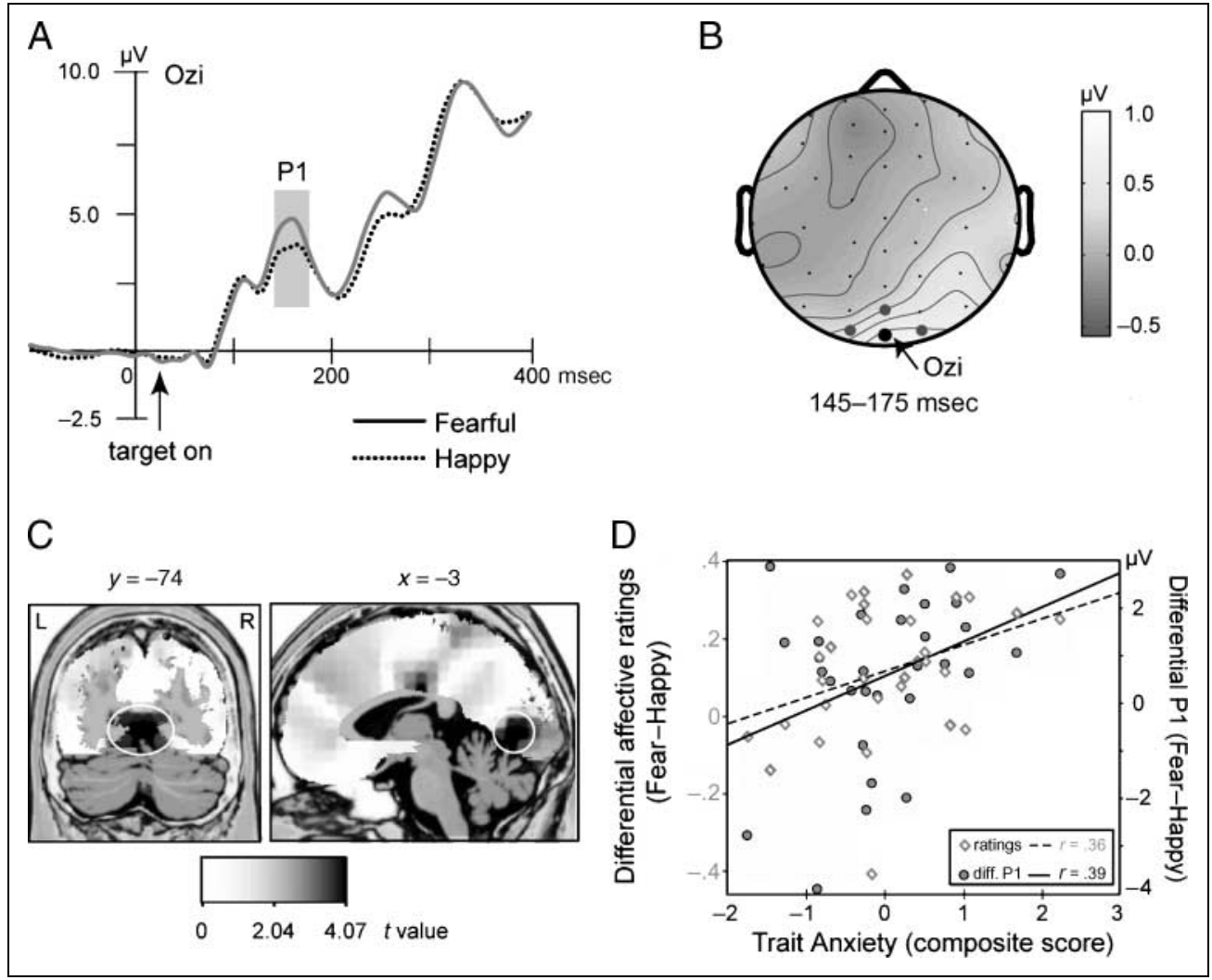

electromagnetic tomography analysis (LORETA) (PascualMarqui et al., 1999; Pascual-Marqui, Michel, \& Lehmann, 1994). Prior studies have produced LORETA estimates consistent with those found in functional hemodynamic imaging studies, reflecting simple visual and auditory processing (Pascual-Marqui et al., 1994), epi- leptic discharges (Worrell et al., 2000), as well as performance in various cognitive tasks (Pizzagalli, Lehmann, Koenig, Regard, \& Pascual-Marqui, 2000; Strik, Fallgatter, Brandeis, \& Pascual-Marqui, 1998). LORETA has also been cross-validated in a study with both ERP and functional magnetic resonance imaging (fMRI), in that
Figure 3. (A) ERP waveforms at a fronto-central location for fearful and happy conditions, with a 100-msec baseline. (B) Topography of differential ERPs (fearful-happy) over the interval from 300 to $400 \mathrm{msec}$. The electrodes where P3 amplitudes were analyzed are marked by large dark dots. In statistical analyses, amplitudes at these electrode locations were grouped (as circled) to reflect frontal, central, and posterior activity. (C) In the LORETA source model, the right posterior parietal region (marked in black and circled on coronal and sagittal brain images) showed enhanced current density for happy than fearful trials at 300-400 msec, collapsed across both TA groups. $t=3.04$ corresponds to $p=.005$.

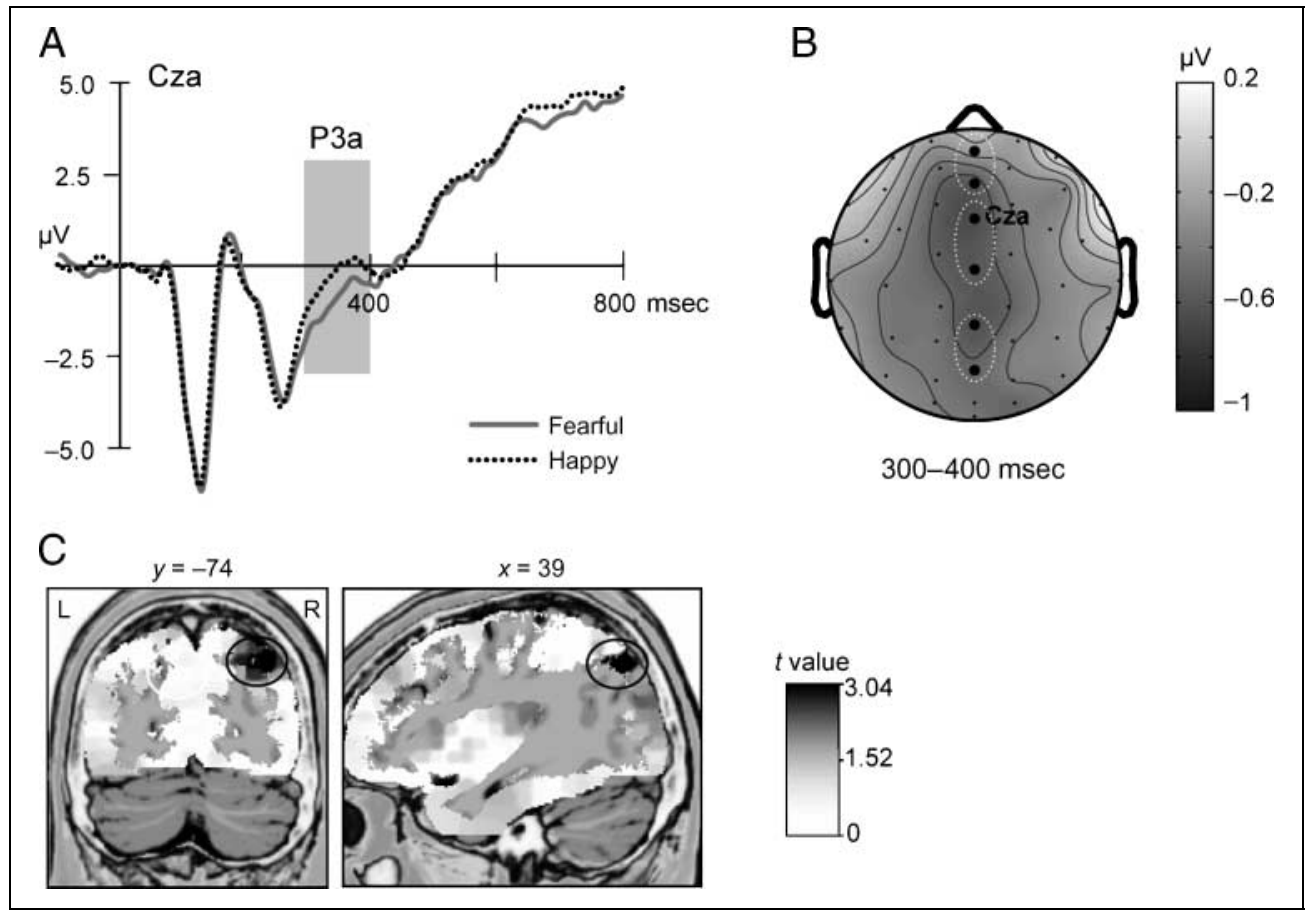


the locations of LORETA generators were remarkably close to those areas identified by fMRI (Pizzagalli et al., 2000; Worrell et al., 2000). Although these methods do not conclusively identify precise anatomical sources of scalp-recorded ERPs, LORETA results are useful for guiding our theorizing about neural mechanisms.

In LORETA, a three-shell spherical head model (Pizzagalli et al., 2000; Pascual-Marqui et al., 1999) is used with electrode coordinates derived from crossregistrations between spherical and realistic head geometry (Towle et al., 1993). The head model has been registered to a standardized stereotactic space (Talairach \& Tournoux, 1988), such that current density in each of 2394 voxels $(7 \times 7 \times 7 \mathrm{~mm})$ in the brain can be estimated (Pourtois et al., 2004; Pizzagalli, Greischar, $\&$ Davidson, 2003). Here, voxelwise $t$ tests were conducted to compare happy and fearful conditions. A cluster was identified if it involved at least three voxels (volume $>1.03 \mathrm{~cm}^{3}$ ) with effects at the level of $p<$ .001 (uncorrected).

\section{RESULTS}

\section{Behavioral Results}

Affective priming was demonstrated by a significant difference in mean ratings for surprise faces preceded by fearful versus happy faces. Ratings for fearful trials tended towards negative affect (higher values) compared to those for happy trials $[F(1,29)=16.51$, $p<.001, \eta^{2}=.33$ ]. Mean ratings were $4.01(S D=$ $.32)$ for fearful trials and $3.90(S D=.31)$ for happy trials. ${ }^{1}$ As predicted, TA exerted a systematic modulation on this priming effect, shown as a significant interaction between prime valence and TA composite scores $[F(1$, $\left.29)=4.22, p<.05, \eta^{2}=.08\right]$. The magnitude of affective priming (fearful-happy) was positively correlated with participants' TA scores $(r=.36, p<.05$; Figure 2D). Further analysis showed a significant correlation between TA and affective ratings in the fearful condition $(r=.36, p<.05)$, but not in the happy condition $(r=.17, p=.35)$.

Reaction time did not differ between the two conditions $[F(1,29)=.52, p=.48]$ or vary with TA $[F(1,29)=$ $1.07, p=.31]$. Mean reaction times were $1263 \mathrm{msec}$ (296) and 1267 msec (302) for fearful and happy trials, respectively.

\section{ERP Results}

Figure 2A illustrates that occipital P1 potentials differed for trials primed by fearful versus happy faces, with larger amplitudes on fearful trials $[F(1,28)=4.23$, $\left.p=.05, \eta^{2}=.11\right]$. Mean P1 amplitudes for the entire sample were $3.41 \mu \mathrm{V}$ (2.96) for fearful trials and $2.82 \mu \mathrm{V}$ (2.98) for happy trials. The scalp topography of this effect is shown in Figure 2B.
Paralleling the behavioral results, this P1 effect was significantly modulated by TA $[F(1,28)=4.93, p<.05$, $\left.\eta^{2}=.14\right]$. As shown in Figure 2D, a positive correlation was observed between the magnitude of P1 differences (fearful-happy) and TA scores $(r=.39, p<.05)$. Similar to the behavioral effect, a positive correlation between P1 and TA appeared in the fearful condition $(r=.38$, $p<.05)$ rather than the happy condition $(r=.15$, $p=.42)$. To underscore the effect of TA, we analyzed ERPs from a group of high-TA individuals and a group of low-TA individuals based on a median split on composite TA scores. In the high-TA subgroup, the mean P1 difference measured $1.05 \mu \mathrm{V}$ (1.40), whereas in the low-TA subgroup, the mean P1 difference measured $0.06 \mu \mathrm{V}(1.87)$.

ERPs also diverged for fearful and happy trials during the P3a interval (300-400 msec, Figure 3A), with a reliable enhancement to happy relative to fearful trials $\left[F(1,28)=5.88, p<.05, \eta_{\text {partial }}{ }^{2}=.17\right]$. The distribution of this difference was fairly broad and was maximal at fronto-central midline locations (Figure 3B). Mean amplitudes averaged across the six midline locations for fearful and happy conditions were $0.72 \mu \mathrm{V}$ (3.84) and $1.23 \mu \mathrm{V}$ (3.80), respectively. This ERP effect was not influenced by TA $[F(1,28)=1.71, p=.20]$ or electrode region $[F(2,56)=.39, p=.68]$.

N2 potentials $(200-300 \mathrm{msec})$ and late positive potentials (400-800 msec) did not exhibit an effect of priming either independently or interactively with TA $\left[F_{\mathrm{S}}<1 ; p \mathrm{~s}>.1\right]$. Finally, there was no significant correlation between the behavioral and ERP indices of priming, suggesting that a complex model beyond merely the mechanisms we tapped here via amplitudes of P1 and P3a potentials is responsible for affective priming.

Influence statistics, $d f_{\text {BETA }}$ values, and Cook's $D$ value (Cohen, Cohen, West, \& Aiken, 2003) were examined in all the above analyses. Results did not reveal any influential cases (at the cutoff of 1), suggesting that effects were not due to any extreme data points. To rule out the influence of individual detection ability of prime features or prime expressions on the effects of interest, we also entered accuracy rates for face/nonface judgments and affect discrimination into regression analyses. We found no evidence that detection ability influenced priming or ERP effects, or their relation with TA $(F \mathrm{~S}<1$, $p s>.44)$, and all the above findings remained essentially the same, after partialing out the contribution of detection variability.

\section{LORETA Results}

\section{P1 Interval (145-175 msec)}

No cluster was identified for the contrast between fearful and happy trials when the entire sample was considered. Given the significant influence of TA found on 
differential P1 potentials, these contrasts were also analyzed separately for the high- and low-TA group. A cluster was thus isolated in bilateral extrastriate cortices in the high-TA group (Figure 2C). This cluster [10 voxels in bilateral lingual gyri, Brodmann's area 18; Montreal Neurology Institute (MNI) coordinates $x, y, z=-3$, $-74,1]$ indicated greater current density for fearful than happy trials $[t(15)=5.25, p<.0001, f=1.31]$.

\section{P3a Interval (300-400 msec)}

No cluster was identified for differential responses for fearful and happy trials at $p<.001$, uncorrected. At a less stringent threshold $(p<.005$ uncorrected), a cluster emerged in the right posterior parietal cortex [3 voxels, Brodmann's areas 7 and 19; MNI coordinates $x, y, z=39,-74,43 ; t(30)=3.61, p=.001 ; f=.97]$, with greater current density for happy than fearful trials (Figure 3C). This effect was equivalent for the high- and low-TA groups.

\section{DISCUSSION}

We demonstrated behavioral and neural correlates of affective priming from unconsciously perceived emotional facial expressions. Fearful and happy prime faces led to valence-consistent biases in affective judgments of ambiguous expressions of surprise. ERP results showed that subliminal primes differentially influenced visual processing at about $145-175 \mathrm{msec}$ after prime onset, most likely arising from neurons in the extrastriate visual cortex. Another ERP effect at 300-400 msec may reflect attentional orienting engendered by the primes. These aspects of emotional processing were further illuminated due to the finding that individual differences in TA played a modulatory role at both behavioral and neural levels.

Because target stimuli were perfectly balanced across the two critical conditions, we can attribute differences in behavior and ERPs to the contrasting features of the subliminal primes. Although affective valence was not strictly the only way in which happy and fearful faces may have differed, we infer that the emotional content of primes mediated these effects because of the significant association between TA and differential responses. It is unlikely that any possible confounding factor that might differentiate the specific happy and fearful primes would be related to TA in this manner.

Another important methodological issue concerns the possibility that participants were aware of the affective expressions of primes. We applied both subjective and objective criteria to assess awareness of facial affect, in that we included verbal reports on participant's phenomenological experiences along with discrimination tasks meant to assess the information participants could extract from the primes. Assessments of participants' awareness of facial affect were applied at the individual level, and the results provided no evidence that affective information from prime faces was consciously perceived. The finding of subliminal priming was further substantiated by analyses that excluded data from the few participants who showed above-chance abilities to discriminate between faces with and without internal facial features.

Unlike most previous studies of similar priming phenomena, this study included personality measures, and thus, highlighted the modulatory role of TA in unconscious affective priming. As predicted, evaluations of facial affect were more susceptible to influences of subliminal priming the higher the individual's tendency to experience anxiety in social and other contexts. This influence of TA was carried by fearful trials. TA was correlated with ratings of surprise faces primed by subliminal fear, but not ratings primed by subliminal happiness. These findings lend direct support to the theory that privileged processing of unconscious threat may contribute to conscious experiences of threat in anxious individuals (Mathews \& Macleod, 1986). Influences of unconscious threat on conscious affective analysis may be especially operative in socially anxious people, giving rise to social interpretation biases (a common symptom of social anxiety), such as when they interpret a neutral expression negatively (Yoon \& Zinbarg, in press; Stopa \& Clark, 2000; Amir, Foa, \& Coles, 1998; also see Clark \& Wells, 1995 for a review). Our recent study with subliminal threat words also examined how TA moderates unconscious threat processing (Li et al., 2007), but we did not examine affective judgments in that study, such that interpretations could not be made concerning negative interpretation bias in anxiety. This current study thus provides novel neural evidence concerning this issue.

ERP recordings revealed two types of neural correlates of affective priming, thereby providing further insights into mechanisms underlying this phenomenon. Analyses of occipital P1 potentials indicated that subliminal affect influenced facial processing as early as 115-145 msec after target-face onset (corresponding to 145-175 msec after prime-face onset), and that this effect was stronger among individuals with higher TA. Paralleling the behavioral findings, this association between TA and P1 enhancement was driven by fearful trials. ERP source modeling with LORETA accorded with previous findings (Pourtois et al., 2004), in that fearful trials in the high-TA group elicited greater current density in the P1 interval in the bilateral extrastriate cortex than did happy trials. The association between TA and enhanced perception of threat accords with findings from a neuroimaging study, wherein high-TA subjects exhibited enhanced cortical activation to subliminal threat in the calcarine cortex and fusiform gyrus (Etkin et al., 2004). The temporal characteristics of this processing, as revealed by the present results, support the conclusion that early 
perceptual analysis is facilitated by subliminal threat, particularly in individuals prone to anxiety. This P1 component could reflect early analysis of both the prime and the target, and we speculate that visual analysis of the two faces may be integrated at this stage. However, definitive evidence on this point would require manipulating the relative timing of prime and target stimuli.

A subcortical colliculo-pulvinar-amygdala pathway may mediate this fast and unconscious perceptual processing. Support for this idea has come from research in animals (LeDoux, 1995) and in humans (de Gelder, Morris, \& Dolan, 2005; Liddell et al., 2005; Morris \& Dolan, 2001; Morris, Öhman, \& Dolan, 1998, 1999, with the exception of Pessoa et al., 2002; also see Pessoa, 2005; Pessoa, Japee, \& Ungerleider, 2005). Enhanced amygdala responses to emotional stimuli are especially prominent in anxious individuals (Etkin et al., 2004; Stein, Goldin, Sareen, Zorrilla, \& Brown, 2002; Rauch et al., 2000; Mathews \& Macleod, 1994). Moreover, a reasonable scenario is that preferential threat processing is due to direct projections from the amygdala to visual regions (Amaral, Price, Pitkanen, \& Carmichael, 1992), which boost responses in the fusiform gyrus and extrastriate cortex (Surguladze et al., 2003; Vuilleumier et al., 2003; Pessoa et al., 2002; also see Phelps, 2006 for a review), an area where P1 is thought to be generated (Pourtois et al., 2004; Gomez Gonzalez et al., 1994; Mangun et al., 1993). Although recent evidence suggests that an enhanced amygdala response can occur to both positive and negative stimuli, provided they are highly arousing (Winston, Gottfried, Kilner, \& Dolan, 2005; Anderson et al., 2003; Small et al., 2003; also see Anderson, 2005; Schimmack \& Derryberry, 2005 for behavioral evidence), our finding of enhanced perception for fear versus happiness in anxious individuals suggests that anxiety could specifically magnify the facilitation of threat perception.

An additional influence of subliminal emotional faces was evident in potentials produced approximately $200 \mathrm{msec}$ after the P1 effects. Similar to previous reports of enlarged P3a potentials to supraliminal positive versus negative stimuli (e.g., Cuthbert et al., 2000), P3a amplitudes were greater for trials primed by happy than fearful faces. This augmentation in P3a was accompanied by concurrent enhancement in current density for happy versus fearful trials in the right posterior parietal area, albeit at a lenient threshold $(p<.005)$. This area approximates the region isolated in an earlier LORETA study where supraliminal liked faces elicited greater current density than did disliked faces (Pizzagalli et al., 2002). Given the putative association between P3a and attention orienting and the link between parietal cortex and attention (Posner \& Dehaene, 1994), especially attention to reward and punishment (Armony \& Dolan, 2002; Critchley, Mathias, \& Dolan, 2001), these data suggest that subliminal affective sensory input can alter attentional orienting to supraliminal targets.
Collectively, the P1 and P3a effects figure nicely into proposals that the environment is scanned for level of threat in an automatic and unconscious fashion (Mathews \& Mackintosh, 1998; Öhman, 1993). Similarly, the ERP data are in keeping with conjectures regarding "default" threat avoidance, wherein people tend to orient away from aversive and toward appetitive stimuli, provided that the threat is insignificant (Mathews \& Mackintosh, 1998; Cacioppo, Gardner, \& Berntson, 1997). Early P1 differences might reflect the action of this hypothesized threat-evaluation system, leading to greater extrastriate activity for threat than nonthreat cues, which may have contributed to the behavioral priming effect on affective evaluation. Yet, threat could not be consciously experienced, perhaps because the primes were too brief, too artificial, and/or too effectively masked by target surprise faces. Accordingly, this effect of primes might not be strong enough to shift the "default" threat-avoidance mode. In this way, surpriseface targets attracted more attention (presumably indexed by P3a differences) when preceded by subliminal positive input than by subliminal negative input. The effect of TA on differential P1 further suggests that the early automatic threat evaluation varies with proneness to anxiety, whereas the later attentional orienting presumably indexed by differential P3a potentials was not modulated by TA status, as threat avoidance may be present regardless of TA status.

At late stages of information processing (400-800 msec), late positive potentials failed to differentiate conditions with fearful versus happy primes, in contrast to a previous report with fearful and happy faces presented in a supraliminal manner (Schupp et al., 2004). We suspect that these late potentials, often associated with conscious cognitive processing, reflect processing of supraliminal targets, but not of subliminal affective information. These results were compatible with the conclusion that subjects were not aware of the prime affect, or of the bias in their affective ratings that was produced by the prime faces. Accordingly, we postulate that subliminal affective information altered early perceptual analyses in the absence of conscious awareness of such an influence. In these circumstances, people can execute the volitional act of evaluating another person, and be conscious of aspects of the decision process and response, without necessarily being aware that their evaluations were systematically modified by affective sensory input.

We speculate further that affective priming may arise as the result of some perceptual integration of prime information and target information. Animal data suggest that backward masking may prevent conscious perception by disrupting interactions between higher and lower parts of the visual processing stream (Lamme, Zipser, \& Spekreijse, 2002; Lamme \& Roelfsema, 2000). Accordingly, when feedback signals concerning the prime face are sent from the inferior temporal cortex 
back to the occipital cortex, these occipital regions are receiving sensory information concerning the target face. Conscious perception of the prime face may fail to occur because of the absence of a synchronized confluence of bottom-up and top-down signals. The conjunction of neural inputs concerning the prime face and the target face, predominated by the latter, may result in a unitary facial representation slightly biased to the affect of the prime. This modified representation can then influence subsequent information processing. In our priming paradigm, conscious perception of fearful and happy faces might be precluded while a unitary image emerges as a surprise face with a tinge of fear or happiness. This slight distortion, perhaps resembling a microexpression of the emotion in the prime face (Ekman, 2003; Ekman \& Friesen, 1969), may give rise to a subtle shift in conscious affective perception in the direction of the affect of the prime face. Electrophysiological signals of enhanced early perception and altered attention orienting, as demonstrated here, may reflect only a fraction of the brain mechanisms that produce affective priming. Many more processes are yet to be uncovered to fully understand this phenomenon.

Personality assessments provided an important, extra dimension to our analysis. Results showed a modulatory role of TA in early visual processing and in the subsequent affective rating, highlighting the importance of taking individual differences into account. By including personality assessments, it is possible to obtain insights into emotion processing that would be lost if every individual was assumed to be the same. The current findings not only show how affective information can bias processing in individuals generally, but also how such processing varies across people. To the extent that a person is prone to anxiety or sensitive to threat, there may be high activation of a threat-surveillance system that captures fleeting threat information so as to bias affective judgments of ambiguous stimuli, which may potentially produce the pervasive negative interpretations and attitudes common in anxiety disorders.

\section{APPENDIX I}

Correlation among Anxiety Indices and Dependent Variables of Interest

\begin{tabular}{|c|c|c|c|c|c|c|}
\hline & \multicolumn{3}{|c|}{ Affective Ratings } & \multicolumn{3}{|c|}{$P 1$} \\
\hline & Fear & Happy & Fear-Happy & Fear & Happy & Fear-Happy \\
\hline BIS & .28 & .17 & .21 & $.38 *$ & .14 & $.41 *$ \\
\hline SPS & $.37 *$ & .14 & $.43 *$ & .30 & .14 & .28 \\
\hline TA & $.36 *$ & .17 & $.36 *$ & $.38 *$ & .15 & $.39 *$ \\
\hline
\end{tabular}

\section{Acknowledgments}

This material is based upon work supported by the National Science Foundation under Grant no. 051880. We thank Paul Whalen and Diego Pizzagalli for fruitful discussions, and Sam Gershman for help with data collection.

Reprint requests should be sent to Wen $\mathrm{Li}$, Northwestern University, 320 E. Superior St., Searle 11-453, Chicago, IL 60611, or via e-mail: wenli@northwestern.edu.

\section{Note}

1. The magnitude of the priming effect in the full group (0.11) was about the same as in the group that excluded all six subjects who could discriminate featureless faces from real faces at any level of confidence (0.11), which was also significant $(p<.01)$.

\section{REFERENCES}

Amaral, D. G., Price, J. L., Pitkanen, A., \& Carmichael, S. T. (1992). Anatomical organization of the primate amygdaloid complex. New York: Wiley-Liss.

Amir, N., Foa, E. B., \& Coles, M. E. (1998). Negative interpretation bias in social phobia. Behaviour Research and Therapy, 36, 945-957.

Anderson, A. K. (2005). Affective influences on the attentional dynamics supporting awareness. Journal of Experimental Psychology: General, 134, 258-281.

Anderson, A. K., Christoff, K., Stappen, I., Panitz, D., Ghahremani, D. G., Glover, G., et al. (2003). Dissociated neural representations of intensity and valence in human olfaction. Nature Neuroscience, 6, 196-202.

Armony, J. L., \& Dolan, R. J. (2002). Modulation of spatial attention by fear-conditioned stimuli: An event-related fMRI study. Neuropsychologia, 40, 817-826.

Beck, A. T., \& Clark, D. A. (1997). An information processing model of anxiety: Reconsidering the role of automatic and strategic processes. Behaviour Research and Therapy, 35, 49-58.

Cacioppo, J. T., Gardner, W. L., \& Berntson, G. G. (1997). Beyond bipolar conceptualizations and measures: The case of attitudes and evaluative space. Personality and Social Psychology Review, 1, 3-25.

Campbell-Sills, L., Liverant, G., \& Brown, T. A. (2004). Psychometric evaluation of the behavioral inhibition/ behavioral activation scales in a large sample of outpatients with anxiety and mood disorders. Psychological Assessment, 16, 244-254.

Carver, C. S., \& White, T. L. (1994). Behavioral inhibition, behavioral activation, and affective responses to impending reward and punishment-The BIS/BAS scales. Journal of Personality and Social Psychology, 67, 319-333.

Clark, D. M., \& Wells, A. (1995). The cognitive model of social phobia. New York: Guilford Press.

Cohen, J., Cohen, P., West, S. G., \& Aiken, L. S. (2003). Applied multiple regression/correlation analysis for the behavioral sciences. Mahwah, NJ: Erlbaum.

Critchley, H. D., Mathias, C. J., \& Dolan, R. J. (2001). Neural activity in the human brain relating to uncertainty and arousal during anticipation. Neuron, 29, 537-545.

Cuthbert, B. N., Schupp, H. T., Bradley, M. M., Birbaumer, N., \& Lang, P. J. (2000). Brain potentials in affective picture processing: Covariation with autonomic arousal and affective report. Biological Psychology, 52, 95-111. 
de Gelder, B., Morris, J. S., \& Dolan, R. J. (2005). Unconscious fear influences emotional awareness of faces and voices. Proceedings of the National Academy of Sciences, U.S.A., 102, 18682-18687.

Dietrich, D. E., Emrich, H. M., Waller, C., Wieringa, B. M., Johannes, S., \& Munte, T. F. (2000). Emotion/ cognition-coupling in word recognition memory of depressive patients: An event-related potential study. Psychiatry Research, 96, 15-29.

Eimer, M., \& Holmes, A. (2002). An ERP study on the time course of emotional face processing. NeuroReport, 13, $427-431$.

Ekman, P. (2003). Emotions revealed: Recognizing faces and feelings to improve communication and emotional life. New York: Times books.

Ekman, P., \& Friesen, W. V. (1969). The repertoire of nonverbal behavior: Categories, origins, usage, and coding. Semiotica, 1, 49-98.

Etkin, A., Klemenhagen, K. C., Dudman, J. T., Rogan, M. T., Hen, R., Kandel, E. R., et al. (2004). Individual differences in trait anxiety predict the response of the basolateral amygdala to unconsciously processed fearful faces. Neuron, 44, 1043-1055.

Fazio, R. H. (2001). On the automatic activation of associated evaluations: An overview. Cognition and Emotion, 15, 115-141.

Fowles, D. C. (1987). Application of a behavioral theory of motivation to the concepts of anxiety and impulsivity. Journal of Research in Personality, 21, 417-435.

Gomez Gonzalez, C. M., Clark, V. P., Fan, S., Luck, S. J., \& Hillyard, S. A. (1994). Sources of attention-sensitive visual event-related potentials. Brain Topography, 7 , 41-51.

Gusnard, D. A., \& Raichle, M. E. (2001). Searching for a baseline: Functional imaging and the resting human brain. Nature Reviews Neuroscience, 2, 685-694.

Halgren, E., \& Marinkovic, K. (1995). Neurophysiological networks integrating buman emotions. Cambridge: MIT Press.

Holmes, A., Vuilleumier, P., \& Eimer, M. (2003). The processing of emotional facial expression is gated by spatial attention: Evidence from event-related brain potentials. Brain Research, Cognitive Brain Research, 16, 174-184.

Johnson, S. L., Turner, R. J., \& Iwata, N. (2003). BIS/BAS levels and psychiatric disorder: An epidemiological study. Journal of Psychopathology and Behavioral Assessment, $25,25-36$.

Johnston, V. S., Miller, D. R., \& Burleson, M. H. (1986). Multiple $\mathrm{P} 3 \mathrm{~s}$ to emotional stimuli and their theoretical significance. Psychophysiology, 23, 684-694.

Keil, A., Moratti, S., Sabatinelli, D., Bradley, M. M., \& Lang, P. J. (2005). Additive effects of emotional content and spatial selective attention on electrocortical facilitation. Cerebral Cortex, 15, 1187-1197.

Kemp-Wheeler, S. M., \& Hill, A. B. (1988). Semantic priming without awareness: Some methodological considerations and replications. Quarterly Journal of Experimental Psychology: Series A, 40, 671-692.

Kenemans, J. L., Verbaten, M. N., Melis, C. J., \& Slangen, J. L. (1992). Visual stimulus change and the orienting reaction: Event-related potential evidence for a two-stage process. Biological Psychology, 33, 97-114.

Killgore, W. D., \& Yurgelun-Todd, D. A. (2005). Social anxiety predicts amygdala activation in adolescents viewing fearful faces. NeuroReport, 16, 1671-1675.

Kim, H., Somerville, L. H., Johnstone, T., Alexander, A. L., \& Whalen, P. J. (2003). Inverse amygdala and medial prefrontal cortex responses to surprised faces. NeuroReport, 14 , 2317-2322

Kim, H., Somerville, L. H., Johnstone, T., Polis, S., Alexander, A. L., Shin, L. M., et al. (2004). Contextual modulation of amygdala responsivity to surprised faces. Journal of Cognitive Neuroscience, 16, 1730-1745.

Lamme, V. A., \& Roelfsema, P. R. (2000). The distinct modes of vision offered by feedforward and recurrent processing. Trends in Neurosciences, 23, 571-579.

Lamme, V. A., Zipser, K., \& Spekreijse, H. (2002). Masking interrupts figure-ground signals in V1. Journal of Cognitive Neuroscience, 14, 1044-1053.

LeDoux, J. E. (1995). Emotion: Clues from the brain. Annual Review of Psychology, 46, 209-235.

Li, W., Zinbarg, R. E., \& Paller, K. A. (2007). Trait anxiety modulates both supraliminal and subliminal threat: Brain potential evidence for both early and late processing influences. Cognitive, Affective, and Behavioral Neuroscience, 7, 25-36.

Liddell, B. J., Brown, K. J., Kemp, A. H., Barton, M. J., Das, P., Peduto, A., et al. (2005). A direct brainstem-amygdalacortical "alarm" system for subliminal signals of fear. Neuroimage, 24, 235-243.

Liddell, B. J., Williams, L. M., Rathjen, J., Shevrin, H., \& Gordon, E. (2004). A temporal dissociation of subliminal versus supraliminal fear perception: An event-related potential study. Journal of Cognitive Neuroscience, 16, 479-486.

Lundqvist, D., Flykt, A., \& Öhman, A. (1998). The Karolinska directed emotional faces. Stockholm: Karolinska Institute, Psychology Section, Department of Clinical Neuroscience.

Mangun, G. R., Hillyard, S. A., \& Luck, S. L. (1993). Electrocortical substrates of visual selective attention. Cambridge: MIT Press.

Mathews, A., \& Mackintosh, B. (1998). A cognitive model of selective processing in anxiety. Cognitive Therapy and Research, 22, 539-560.

Mathews, A., \& Macleod, C. (1986). Discrimination of threat cues without awareness in anxiety-states. Journal of Abnormal Psychology, 95, 131-138.

Mathews, A., \& Macleod, C. (1994). Cognitive approaches to emotion and emotional disorders. Annual Review of Psychology, 45, 25-50.

Mathews, A., Yiend, J., \& Lawrence, A. D. (2004). Individual differences in the modulation of fear-related brain activation by attentional control. Journal of Cognitive Neuroscience, 16, 1683-1694.

Mattick, R. P., \& Clarke, J. C. (1998). Development and validation of measures of social phobia scrutiny fear and social interaction anxiety. Behaviour Research and Therapy, 36, 455-470.

Mogg, K., \& Bradley, B. P. (1998). A cognitive-motivational analysis of anxiety. Behaviour Research and Therapy, 36, 809-848.

Morris, J. S., \& Dolan, R. J. (2001). The amygdala and unconscious fear processing. Oxford: Oxford University Press.

Morris, J. S., Öhman, A., \& Dolan, R. J. (1998). Conscious and unconscious emotional learning in the human amygdala. Nature, 393, 467-470.

Morris, J. S., Öhman, A., \& Dolan, R. J. (1999). A subcortical pathway to the right amygdala mediating "unseen" fear. Proceedings of the National Academy of Sciences, U.S.A., 96, 1680-1685.

Murphy, S. T., \& Zajonc, R. B. (1993). Affect, cognition, and awareness: Affective priming with optimal and suboptimal stimulus exposures. Journal of Personality and Social Psychology, 64, 723-739. 
Öhman, A. (1988). Nonconscious control of autonomic responses: A role for Pavlovian conditioning? Biological Psychology, 27, 113-135.

Öhman, A. (1993). Fear and anxiety as emotional phenomena. New York: Guilford Press.

Öhman, A. (2005). The role of the amygdala in human fear: Automatic detection of threat. Psychoneuroendocrinology, 30, 953-958.

Öhman, A., \& Soares, J. J. (1998). Emotional conditioning to masked stimuli: Expectancies for aversive outcomes following nonrecognized fear-relevant stimuli. Journal of Experimental Psychology: General, 127, 69-82.

Pascual-Marqui, R. D., Lehmann, D., Koenig, T., Kochi, K., Merlo, M. C., Hell, D., et al. (1999). Low resolution brain electromagnetic tomography (LORETA) functional imaging in acute, neuroleptic-naive, first-episode, productive schizophrenia. Psychiatry Research, 90, 169-179.

Pascual-Marqui, R. D., Michel, C. M., \& Lehmann, D. (1994). Low resolution electromagnetic tomography: A new method for localizing electrical activity in the brain. International Journal of Psychophysiology, 18, 49-65.

Pessoa, L. (2005). To what extent are emotional visual stimuli processed without attention and awareness? Current Opinion in Neurobiology, 15, 188-196.

Pessoa, L., Japee, S., \& Ungerleider, L. G. (2005). Visual awareness and the detection of fearful faces. Emotion, 5, 243-247.

Pessoa, L., McKenna, M., Gutierrez, E., \& Ungerleider, L. G. (2002). Neural processing of emotional faces requires attention. Proceedings of the National Academy of Sciences, U.S.A., 99, 11458-11463.

Phan, K. L., Fitzgerald, D. A., Nathan, P. J., \& Tancer, M. E. (2006). Association between amygdala hyperactivity to harsh faces and severity of social anxiety in generalized social phobia. Biological Psychiatry, 59, 424-429.

Phelps, E. A. (2006). Emotion and cognition: Insights from studies of the human amygdala. Annual Review of Psychology, 57, 27-53.

Pizzagalli, D., Lehmann, D., Koenig, T., Regard, M., \& Pascual-Marqui, R. D. (2000). Face-elicited ERPs and affective attitude: Brain electric microstate and tomography analyses. Clinical Neurophysiology, 111, 521-531.

Pizzagalli, D. A., Greischar, L. L., \& Davidson, R. J. (2003). Spatio-temporal dynamics of brain mechanisms in aversive classical conditioning: High-density event-related potential and brain electrical tomography analyses. Neuropsychologia, 41, 184-194.

Pizzagalli, D. A., Lehmann, D., Hendrick, A. M., Regard, M., Pascual-Marqui, R. D., \& Davidson, R. J. (2002). Affective judgments of faces modulate early activity (approximately $160 \mathrm{~ms}$ ) within the fusiform gyri. Neuroimage, 16, 663-677.

Posner, M. I., \& Dehaene, S. (1994). Attentional networks. Trends in Neurosciences, 17, 75-79.

Pourtois, G., Grandjean, D., Sander, D., \& Vuilleumier, P. (2004). Electrophysiological correlates of rapid spatial orienting towards fearful faces. Cerebral Cortex, 14, 619-633.

Raichle, M. E., \& Gusnard, D. A. (2005). Intrinsic brain activity sets the stage for expression of motivated behavior. Journal of Comparative Neurology, 493, 167-176.

Rauch, S. L., Whalen, P. J., Shin, L. M., McInerney, S. C., Macklin, M. L., Lasko, N. B., et al. (2000). Exaggerated amygdala response to masked facial stimuli in posttraumatic stress disorder: A functional MRI study. Biological Psychiatry, 47, 769-776.

Schimmack, U., \& Derryberry, D. (2005). Attentional interference effects of emotional pictures: Threat, negativity, or arousal? Emotion, 5, 55-66.

Schupp, H. T., Cuthbert, B. N., Bradley, M. M., Cacioppo, J. T., Ito, T., \& Lang, P. J. (2000). Affective picture processing: The late positive potential is modulated by motivational relevance. Psychophysiology, 37, 257-261.

Schupp, H. T., Junghofer, M., Weike, A. I., \& Hamm, A. O. (2003). Attention and emotion: An ERP analysis of facilitated emotional stimulus processing. NeuroReport, 14, 1107-1110.

Schupp, H. T., Öhman, A., Junghofer, M., Weike, A. I., Stockburger, J., \& Hamm, A. O. (2004). The facilitated processing of threatening faces: An ERP analysis. Emotion, 4, 189-200.

Simon-Thomas, E. R., Role, K. O., \& Knight, R. T. (2005). Behavioral and electrophysiological evidence of a right hemisphere bias for the influence of negative emotion on higher cognition. Journal of Cognitive Neuroscience, 17, 518-529.

Small, D. M., Gregory, M. D., Mak, Y. E., Gitelman, D., Mesulam, M. M., \& Parrish, T. (2003). Dissociation of neural representation of intensity and affective valuation in human gustation. Neuron, 39, 701-711.

Smith, N. K., Cacioppo, J. T., Larsen, J. T., \& Chartrand, T. L. (2003). May I have your attention, please: Electrocortical responses to positive and negative stimuli. Neuropsychologia, 41, 171-183.

Spielberger, C. D. (1983). Manual for the state-trait anxiety inventory (STAI). Palo Alto, CA: Consulting Psychologists Press.

Stein, M. B., Goldin, P. R., Sareen, J., Zorrilla, L. T., \& Brown, G. G. (2002). Increased amygdala activation to angry and contemptuous faces in generalized social phobia. Archives of General Psychiatry, 59, $1027-1034$.

Stopa, L., \& Clark, D. M. (2000). Social phobia and interpretation of social events. Behaviour Research and Therapy, 38, 273-283.

Strik, W. K., Fallgatter, A. J., Brandeis, D., \& Pascual-Marqui, R. D. (1998). Three-dimensional tomography of event-related potentials during response inhibition: Evidence for phasic frontal lobe activation. Electroencephalography and Clinical Neurophysiology, 108, 406-413.

Surguladze, S. A., Brammer, M. J., Young, A. W., Andrew, C., Travis, M. J., Williams, S. C., et al. (2003). A preferential increase in the extrastriate response to signals of danger. Neuroimage, 19, 1317-1328.

Talairach, J., \& Tournoux, P. (1988). Co-planar stereotaxic atlas of the buman brain. New York: Thieme.

Taylor, J. A. (1953). A personality scale of manifest anxiety. Journal of Abnormal and Social Psychology, 48, 285-290.

Towle, V. L., Bolanos, J., Suarez, D., Tan, K., Grzeszczuk, R., Levin, D. N., et al. (1993). The spatial location of EEG electrodes: Locating the best-fitting sphere relative to cortical anatomy. Electroencephalography and Clinical Neurophysiology, 86, 1-6.

Vuilleumier, P., Armony, J. L., Driver, J., \& Dolan, R. J. (2003). Distinct spatial frequency sensitivities for processing faces and emotional expressions. Nature Neuroscience, 6 , 624-631.

West, S. G., Aiken, L. S., \& Krull, J. L. (1996). Experimental personality designs: Analyzing categorical by continuous variable interactions. Journal of Personality, 64, 1-48. 
Whalen, P. J., Rauch, S. L., Etcoff, N. L., McInerney, S. C., Lee, M. B., \& Jenike, M. A. (1998). Masked presentations of emotional facial expressions modulate amygdala activity without explicit knowledge. Journal of Neuroscience, 18, 411-418.

Williams, J. M. G., Watts, F. N., MacLeod, C., \& Mathews, A. (1997). Cognitive psychology and emotional disorders (2nd ed.). Chichester: Wiley.

Williams, L. M., Liddell, B. J., Rathjen, J., Brown, K. J., Gray, J., Phillips, M., et al. (2004). Mapping the time course of nonconscious and conscious perception of fear: An integration of central and peripheral measures. Human Brain Mapping, 21, 64-74.
Winston, J. S., Gottfried, J. A., Kilner, J. M., \& Dolan, R. J. (2005). Integrated neural representations of odor intensity and affective valence in human amygdala. Journal of Neuroscience, 25, 8903-8907.

Worrell, G. A., Lagerlund, T. D., Sharbrough, F. W., Brinkmann, B. H., Busacker, N. E., Cicora, K. M., et al. (2000). Localization of the epileptic focus by low-resolution electromagnetic tomography in patients with a lesion demonstrated by MRI. Brain Topography, 12, 273-282.

Yoon, L. K., \& Zinbarg, R. E. (in press). Threat is in the eye of the beholder: Social anxiety and the interpretation of ambiguous facial expressions. Behaviour Research and Therapy. 